\title{
A Nitrate-Induced frq-Less Oscillator in Neurospora crassa
}

\author{
Melinda K. Christensen, ${ }^{*}$ Grete Falkeid, ${ }^{*}$ Jennifer J. Loros, ${ }^{\dagger}$ Jay C. Dunlap, ${ }^{\ddagger}$ \\ Cathrine Lillo," and Peter Ruoff,"1 \\ "Stavanger University College, School of Science and Technology, Stavanger, Norway; \\ Departments of ${ }^{\dagger}$ Biochemistry and ${ }^{\ddagger}$ Genetics, Dartmouth Medical School, Hanover, NH, USA
}

Abstract When nitrate is the only nitrogen source, Neurospora crassa's nitrate reductase (NR) shows endogenous oscillations in its nitrate reductase activity (NRA) on a circadian time scale. These NRA oscillations can be observed in darkness or continuous light conditions and also in a $f r q^{9}$ mutant in which no functional FRQ protein is formed. Even in a white-collar-1 knockout mutant, NRA oscillations have been observed, although with a highly reduced amplitude. This indicates that the NRA oscillations are not a simple output rhythm of the whitecollar-driven frq oscillator but may be generated by another oscillator that contains the nit-3 autoregulatory negative feedback loop as a part. In this negative feedback loop, a product in the reaction chain catalyzed by nitrate reductase, probably glutamine, induces repression of the nitrate reductase gene and thus downregulates its own production. This is the first example of an endogenous, nutritionally induced daily rhythm with known molecular components that is observed in the absence of an intact FRQ protein.

Key words Neurospora crassa, circadian rhythm, nitrate reductase, oscillatory feedback loops, multiple oscillators, frq-less oscillator (FLO)

Circadian rhythms play central roles in the daily and seasonal adaptation of organisms to their environment (Bünning, 1963; Dunlap et al., 2003; Edmunds, 1988). Considerable progress has been made by using model organisms such as Drosophila (Hall, 1998; Young, 1998) and Neurospora (Davis, 2000; Lakin-Thomas et al., 1990; Loros and Dunlap, 2001; Nakashima and Onai, 1996) to identify and understand the molecular processes responsible for these rhythms. For the organisms investigated so far, all circadian "pacemakers" have been found to be composed of transcriptional-translational negative feedback loops in which clock proteins inhibit their own expression (Dunlap, 1999). In Neurospora crassa, the negative feedback loop of the frequency (frq) gene
(Aronson et al., 1994b) has been found to be a central component in the organism's circadian clock (Loros and Dunlap, 2001), influencing properties such as temperature and $\mathrm{pH}$ compensation (Ruoff et al., 2000). In $f r q$ null strains (e.g., $f r q^{9}$ ), no functional FRQ protein is made, and unlike in wild-type strains, no immediate sporulation rhythm is observed (Loros and Feldman, 1986). The fact, however, that rhythmic conidiation in $\mathrm{frq}^{9}$ (or other frq-less strains) may appear after several days indicates that in the absence of the frq gene, other oscillatory states, termed frqless oscillators (FLOs; Granshaw et al., 2003; LakinThomas and Brody, 2000, in press; McWatters et al., 1999; Merrow et al., 1999), may be formed after the system has passed an "induction period," similar to

1. To whom all correspondence should be addressed: Stavanger University College, School of Science and Technology, P.O. Box 8002 Ullandhaug, 4068 Stavanger, Norway; e-mail: peter.ruoff@tn.his.no.

JOURNAL OF BIOLOGICAL RHYTHMS, Vol. 19 No. 4, August 2004 280-286

DOI: $10.1177 / 0748730404265532$

(C) 2004 Sage Publications 
what is observed in chemical oscillators (Field and Burger, 1985).

In the model plant Arabidopsis thaliana, several genes are known to regulate circadian rhythms, but their functions within a central oscillator have not been established (McClung, 2001). It has been argued (Lillo et al., 2001) that circadian rhythms and their functionality can be perceived without postulating a central molecular chronometer. For example, the light-induced circadian nitrate reductase (NR) rhythm observed in several higher plants (Deng et al., 1989; Lillo, 1984; Lillo and Ruoff, 1989; McClung and Kay, 1994) can be understood as the result of an autonomous negative feedback loop in which probably glutamine, a product in the reaction chain initialized by NR, inhibits transcription of the NR gene (Lillo et al., 2001). Because higher plant and Neurospora NRs show similar features, including negative feedback inhibition by glutamine ( $\mathrm{Fu}$ and Marzluf, 1988; Marzluf, 1997), we wondered whether N. crassa nitrate reductase activity (NRA) might also show circadian oscillations and, if so, to what extent an NRA rhythm might be under the control of the frq oscillator. In this report, we show that NRA oscillations in Neurospora occur on a 24-h time scale both under light and dark conditions when nitrate is the only nitrogen source. The NRA rhythm is also found in a $\mathrm{fr}^{9}$ mutant and is thus independent of a functional FRQ protein. Even in a $w c-1$ knockout mutant $\left(w c-1^{k o}\right)$, we have observed NRA oscillations, although with a highly reduced amplitude.

\section{MATERIALS AND METHODS}

The N. crassa strains $b d f r q^{+}$and $b d f r q^{9}$ (both mating type A) were obtained from the Fungal Genetic Stock Center (http://www.fgsc.net). In all experiments, ammonium-free Vogel's medium (Vogel, 1956) was used. A $10 \times$ medium was prepared by replacing the normally used $\mathrm{NH}_{4} \mathrm{NO}_{3}$ by $\mathrm{NaNO}_{3}$, such that the nitrate concentration in the $1 \times$ solution was $25 \mathrm{mM}$. In the trace element solution, the normally used $\left(\mathrm{NH}_{4}\right)_{2} \mathrm{Fe}\left(\mathrm{SO}_{4}\right)_{2}$ was replaced by an equimolar amount of $\mathrm{FeSO}_{4} \bullet 7 \mathrm{H}_{2} \mathrm{O}$. Inoculation of approximately $1 \times 10^{8}$ conidia/L was performed in Petri dishes $(90-\mathrm{mm}$ diameter) containing $20 \mathrm{~mL}$ of $1 \times$ Vogel's medium with $2 \%$ sucrose, and the culture was exposed to white fluorescent light $\left(25 \mu \mathrm{mol} \mathrm{s}^{-1} \mathrm{~m}^{-2}\right)$ at $30^{\circ} \mathrm{C}$. After $36 \mathrm{~h}$ of continuous light exposure, mycelial discs were cut out with a cork borer (1-cm diameter), and Petri dishes containing $20 \mathrm{~mL} 1 \times$ Vogel's medium and low sucrose $(0.005 \%)$ were loaded with 3 mycelial discs each. The Petri dishes with the discs were then placed in darkness at $25^{\circ} \mathrm{C}$. Two time series with a 12 -h time difference were prepared by inoculating series 1 and 2 at $0800 \mathrm{~h}$ and $2000 \mathrm{~h}$, respectively, on day 1 . Harvesting of the mycelial discs started on day 3 and continued over the following days from $0800 \mathrm{~h}$ until $2000 \mathrm{~h}$ with 4-h intervals. Harvesting in dark or light conditions was done by gently placing the discs on filter paper to remove excess liquid. Each set of discs was wrapped in aluminum foil, rapidly frozen in liquid nitrogen, and stored at $-70^{\circ} \mathrm{C}$.

The slightly modified nitrate reductase activity assay (Lillo, 1983) was performed as follows: $1 \mathrm{mLice}-$ cold extraction buffer (0.1 M HEPES [pH 7.5], $1 \mathrm{mM}$ EDTA, 3\% (w/v) polyvinylpolypyrrolidone, $7 \mathrm{mM}$ cysteine) was added to a 3-mL precooled glass homogenizer along with the mycelial discs, which then were rapidly homogenized on ice. In a $2-\mathrm{mL}$ reaction tube, $650 \mu \mathrm{L}$ of assay buffer (50 mM HEPES-KOH [pH 7.5], 2 $\mathrm{mM} \mathrm{KNO}_{3}, 200 \mu \mathrm{MNADPH}, 2 \mathrm{mM}$ EDTA) was kept at $30{ }^{\circ} \mathrm{C}$, and $100 \mu \mathrm{L}$ of the extract was added and vortexed. After $10 \mathrm{~min}$ at $30{ }^{\circ} \mathrm{C}, 700 \mu \mathrm{L}$ of the colordeveloping reagent $(1 \%(\mathrm{w} / \mathrm{v})$ sulfanilamide and $0.02 \%(\mathrm{w} / \mathrm{v}) \mathrm{N}-(1-$ naphtyl)-ethylenediamine dihydrochloride in $1 / 10$ diluted $\mathrm{HCl}$ ) was added and vortexed again. Tubes were centrifuged for $30 \mathrm{~min}$ at $12,000 \mathrm{rpm}$, and the amount of nitrite formed was measured as the absorbance at $540 \mathrm{~nm}$. For each extract, the NRA assay was performed in triplicate.

Amounts of total protein in extracts were measured in duplicate using the Bio-Rad Protein Assay with IgG as a standard. NRA levels are given in $\mu$ mol-formed $\mathrm{NO}_{2}^{-}$per mg protein per hour. The NRA rhythm was analyzed by fitting the average NRA levels from repeated time courses to a variable-amplitude square sinus function $g(t)$,

$$
g(t)=\lambda_{1}+\lambda_{2} e^{-\lambda_{3} \cdot t} \sin ^{2}((\pi / p)+\phi),
$$

using the program KaleidaGraph (Synergy Software, Reading, MA). The $\lambda_{i} \mathrm{~s}$, as well as $P$ (period) and $\phi$, are adjustable parameters, and $t$ is time.

\section{RESULTS AND DISCUSSION}

Figure 1A,B shows the observed NRA rhythm of $b d$ and $f r q^{9}$ mutants in continuous darkness (DD). Because N. crassa NR is photoactivated like plant NR 

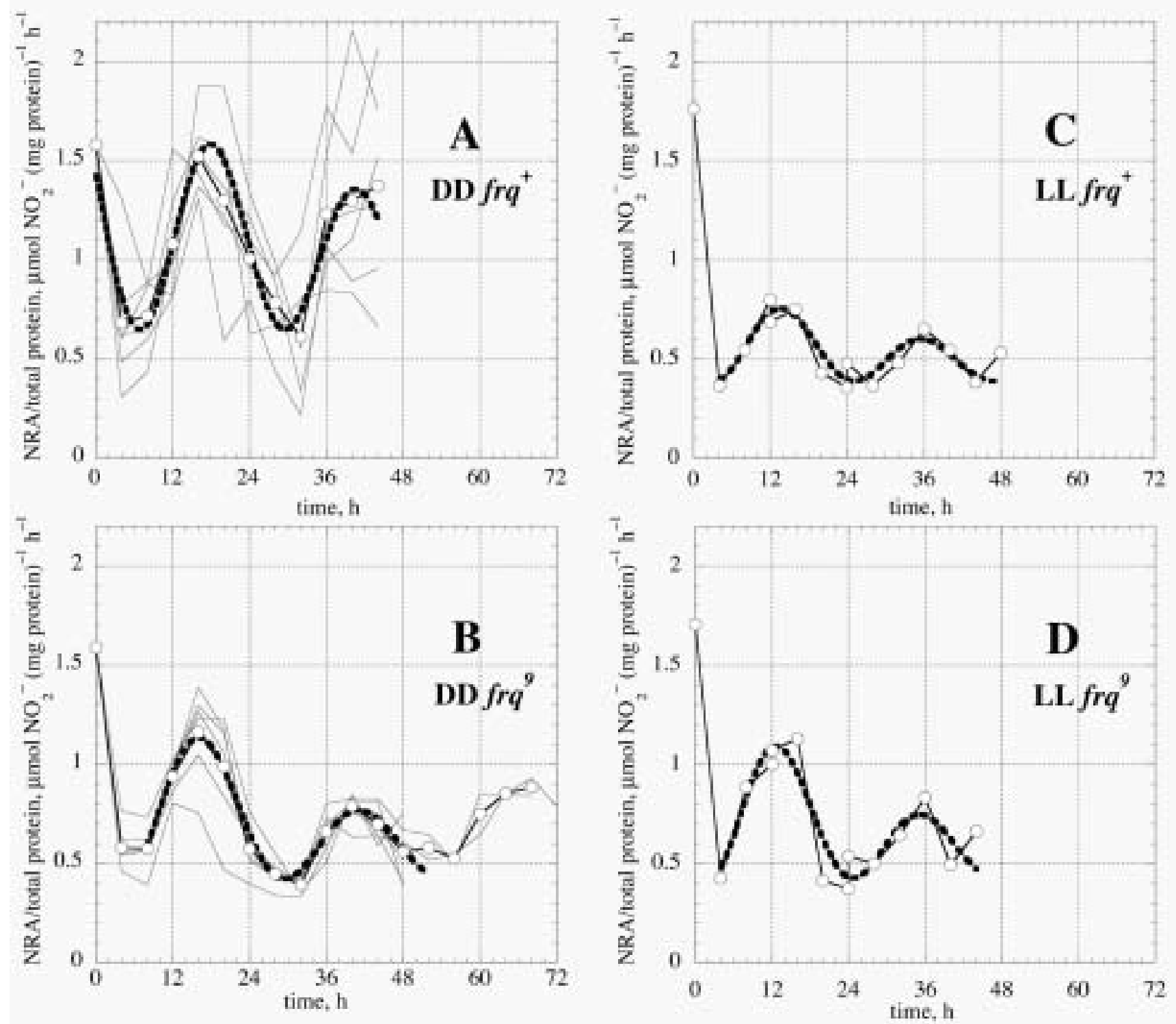

Figure 1. Nitrate reductase activity (NRA) shows endogenous oscillations with a period length of approximately $24 \mathrm{~h}$ in $\mathrm{DD}$, in $L L$, and in the absence of a functional FRQ protein. (A, B) NRA rhythm in DD of (A) $f r q^{+}(n=6)$ and (B) $f r q^{9}\left(n=6\right.$ for $0-48 \mathrm{~h} ; n=2 \mathrm{for} 0-72 \mathrm{~h}$ ) at $25^{\circ} \mathrm{C}$. Time $t=0$ represents the transition from LL with high sucrose to DD with low sucrose. (C, D) Examples of NRA rhythm in LL of $(C) f r q^{+}(n=1)$ and (D) $f r q^{9}(n=1)$ at $25^{\circ} \mathrm{C}$. Time $t=0$ represents the transition in which discs were transferred from high sucrose to low sucrose. Open circles in (A, B) represent average NRA/total protein values. The average values at $t=0$ for (A) and (B) were $1.58 \pm 0.41$ and $1.59 \pm 0.22 \mu \mathrm{mol} \mathrm{NO}-{ }_{2}^{-}(\mathrm{mg}$ protein) ${ }^{-1} h^{-1}$, respectively. Thick dashed lines are curve fits of equation (1) to the averaged experimental data; $R$-values of the curve fits: (A) $0.9328,(B) 0.9708,(C) 0.8880$, and (D) 0.8966 .

(Ninnemann, 1997; Roldán and Butler, 1980) and because plant NRA rhythms are generally light induced (Deng et al., 1989; Lillo, 1984, 1993; Lillo and Ruoff, 1989; McClung and Kay, 1994), we tested whether the Neurospora NRA rhythm may also occur under continuous light (LL) conditions. LL does not abolish the NRA rhythm, as the results in Figure 1C,D show, in contrast to Neurospora's sporulation rhythm and FRQ oscillations (Collett et al., 2002). In both DD and LL, the NRA period length is about $24 \mathrm{~h}$, but in LL (+ low glucose), the rhythm is phase advanced compared to DD. Because the NRA oscillations are also found in $f r q^{9}$, which is a frq null allele (Aronson et al., 1994a), the NRA rhythm is not an output rhythm of the frq oscillator but is self-sustained. That NR oscillations can persist even in the absence of a functional white- 


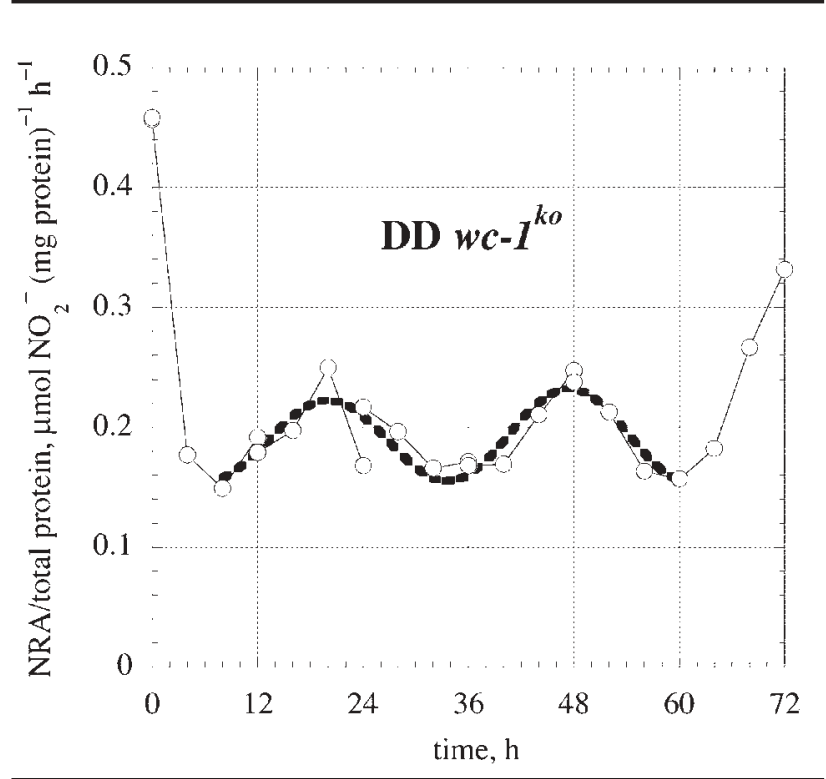

Figure 2. Observed nitrate reductase activity (NRA) oscillations in the absence of a functional white-collar complex $(n=1)$. Note the strongly reduced amplitude and the slight phase delay in the NRA rhythm onset in $w c-1^{k o}$ compared with the NRA oscillations in $f r q^{+}$and $f r q^{9}$ (Fig. 1). Dashed line is the curve fit of equation (1) to the experimental data; $R$-value: 0.8612 . $\mathrm{T}=25^{\circ} \mathrm{C}$. Time $t=0$ represents the transition from LL with high sucrose to DD with low sucrose.

collar complex (WCC) is indicated by results using a $w c-1^{k o}$ mutant. In this mutant, light sensing and frq and other gene transcriptions through the WCC are abolished (Lee et al., 2003). NRA oscillations were observed in this mutant, although with a strongly reduced amplitude (Fig. 2). However, this result is somewhat preliminary because from four independent experiments, oscillations were seen in only two of the cases (one shown in Fig. 2). In the other two cases, it was difficult to judge whether there was a rhythm. We do not know the reason for the large reduction in NRA amplitude and levels in $w c-1^{k 0}$. It may be related to the loss of the light sensor WC-1 (Cheng et al., 2003; Froehlich et al., 2002) or may indicate that WCCdependent processes have an influence on nit-3 transcription and/or translation. Although NR contains a blue light absorbing flavin, which may affect the influence of light on the NR oscillator, the suggested role that NR is a blue light receptor (Ninnemann, 2001) is still not clearly established (Belozerskaya et al., 1982).

Compared with DD, the LL NRA rhythm is phase advanced by 4 to $6 \mathrm{~h}$ (Figs. 1, 3A). The cause for the phase advance in LL lies in an approximately twice as rapid decrease of NRA after the transfer to lowsucrose light conditions (Fig. 3B), while the time interval between the first NRA minimum and the fol- lowing maximum appears unaltered in DD and LL $(\approx 10 \mathrm{~h})$.

In the absence of favored nitrogen sources such as ammonium or glutamine, Neurospora readily takes up nitrate. Utilization of nitrate requires de novo synthesis of nitrate reductase and nitrite reductase, as well as nitrogen derepression and specific induction by nitrate ion (Marzluf, 1997). Glutamine, a downstream reaction product of nitrate reductase, appears to be the critical metabolite, which represses transcription of nitrate reductase (Premakumar et al., 1979). The actual repression mechanism is not completely understood. Glutamine somehow activates the key regulator NMR (nitrogen metabolite regulation), which binds directly to NIT2, which is one of the two recognized positive regulators of nitrate reductase transcription (Fu and Marzluf, 1988; Marzluf, 1997; Pan et al., 1997). Thus, in the presence of only ammonium (or glutamine), the NR oscillator is not expressed, but (in $\mathrm{frq}^{+}$) the FRQ/ WCC-driven circadian conidiation rhythm should still be operative, probably even in a nit-3 knockout mutant.

Similar to what has been observed for higher plants (Lillo et al., 2001), the regulation circuit governing nitrate utilization in Neurospora (Fig. 4) is also a negative feedback loop, which, like in higher plants and algae, may be capable of acting as an autonomous daily rhythm generator. The inactivation and turnover of Neurospora NR and its mRNA have been found to be fairly rapid processes (Okamoto et al., 1991; Sorger et al., 1978; Walls et al., 1978). As for the FRQ negative feedback loop (Ruoff et al., 1999), the degradation of NR and its mRNA may play a role in defining the period length of the NR oscillator and its temperature behavior. In Gonyaulax, nitrate had a profound effect on the circadian rhythm, affecting amplitude, phase, and period (Roenneberg and Rehman, 1996).

It is intriguing that in Neurospora, an additional NR oscillator (besides the frq oscillator) comes into play when environmental conditions require assimilation of nitrate as the only nitrogen source. Interestingly, both FRQ (Garceau et al., 1997) and NRA (Fig. 1) peak at approximately the same phase relative to a light-todark transfer. In fact, microarray analyses (Correa et al., 2003; Nowrousian et al., 2003) show that the majority of rhythmically expressed Neurospora genes $(2.7 \%-5.9 \%)$ peak at approximately the same phase with an increased transcriptional/metabolic activity during late night to early morning.

Because under nitrate-growing conditions, all assimilated nitrogen goes through the nitrate 

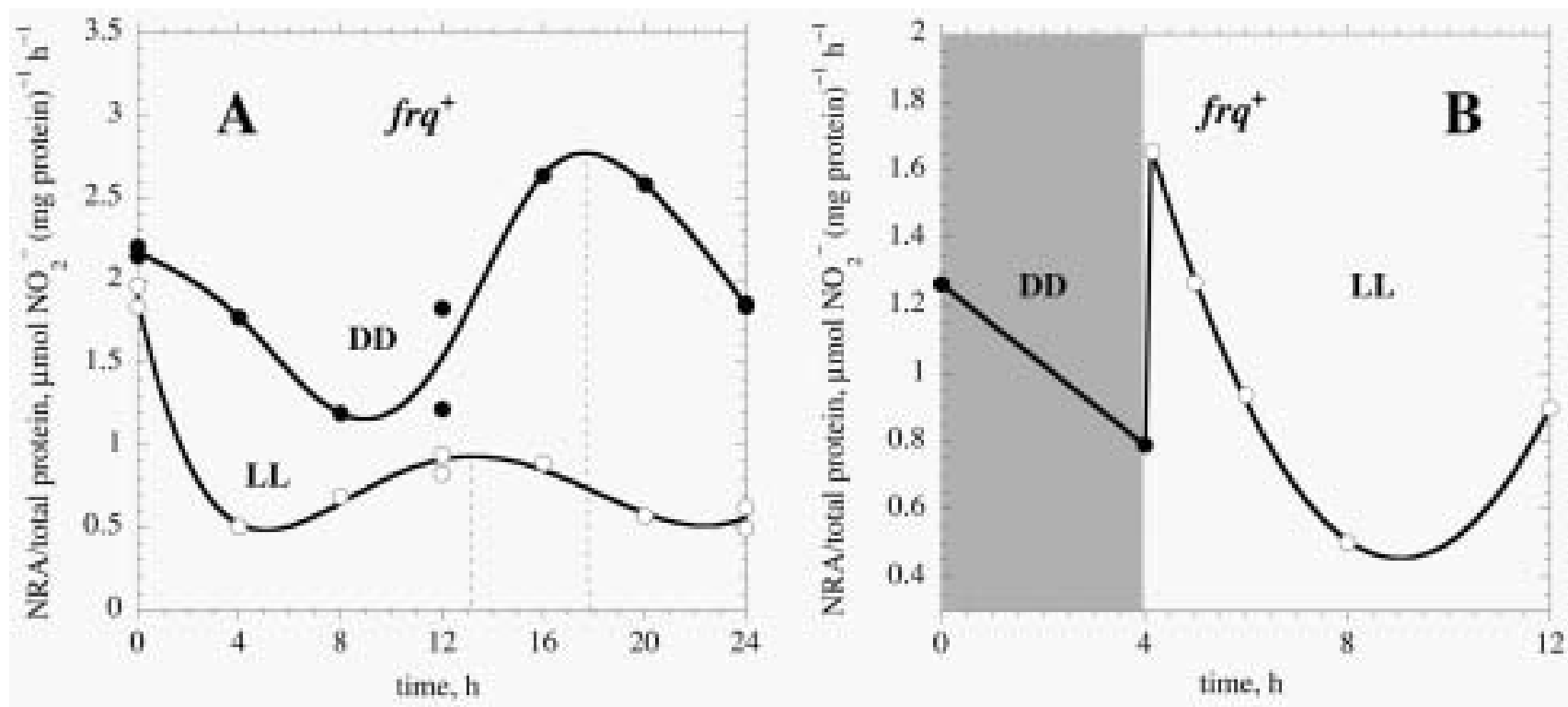

Figure 3. Kinetics of nitrate reductase activity (NRA) in DD and LL during the induction period. Time $t=0$ represents the transition from LL with high sucrose to DD with low sucrose. (A) $\mathrm{frq}^{+}$mycelium discs that were cut out from the same culture (mycelium) and then tested in parallel under DD and LL conditions show a more rapid approach to the oscillatory state in LL than in DD. As a result, the LL rhythm shows a phase advance compared to the DD rhythm. (B) Effect of DD $\rightarrow$ LL transition on $f r q^{+}$NRA. After a rapid light-induced increase, the NRA decreases more rapidly in LL than previously in DD. $\mathrm{T}=25^{\circ} \mathrm{C}$.

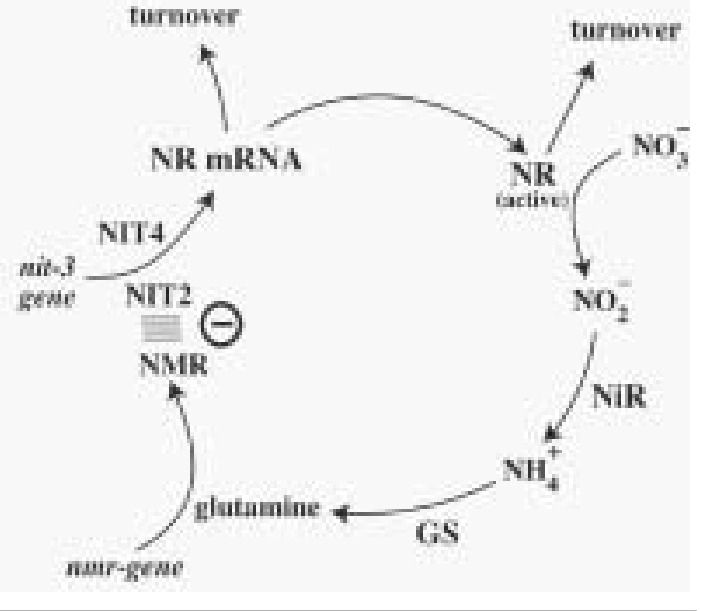

Figure 4. A simplified scheme of nitrate assimilation and the nitrate reductase (NR) negative feedback loop in Neurospora crassa. NIT2 and NIT4 are positive regulators, which are required for the transcription and regulation of the nit-3 gene (the structural gene of NR). Active NR catalyzes the reduction of nitrate to nitrite, which is reduced to ammonium (catalyzed by nitrite reductase $[\mathrm{NiR}]$ ) and further incorporated into glutamine (catalyzed by glutamine synthetase [GS]). Due to a not completely understood mechanism, glutamine activates the negative regulator NMR (nitrogen metabolite regulation), which binds directly to NIT2 and inhibits transcription of nit-3 (Pan et al., 1997; Marzluf, 1997). reductase pathway, the NRA oscillations can be considered as an indicator for Neurospora's metabolic (nitrogen) activity. Although a yet unknown frqindependent oscillator that drives the NRA oscillations could always be postulated, it seems equally reasonable to consider that NR is part of an autonomous oscillator defined partly or wholly by the NR negative feedback loop (Fig. 4). Such an oscillator would regulate nitrate uptake and assimilation to ensure an increased metabolic/transcriptional activity during late night to early morning (Correa et al., 2003; Nowrousian et al., 2003). It is tempting to speculate that other control processes-for example, the sulfur regulatory circuit (Kumar and Paietta, 1995; Perkins et al., 2001)—could be regulated in a similar circadiantype fashion, where positive and negative elements (CYS3 and SCON1/SCON2, respectively) may play similar dynamic roles as the positive and negative regulators in the frq or nit-3 negative feedback loops.

Whether Neurospora's nitrate reductase loop (Fig. 4) can autonomously generate true circadian oscillations, including temperature compensation, or may be part of a network of multiple oscillators (Correa et al., 2003) will be the subject of subsequent work. 


\section{ACKNOWLEDGMENTS}

We thank Stuart Brody, Patricia Lakin-Thomas, Ludger Rensing, and members of our laboratories for comments. We acknowledge support from the Stavanger University Fund, Stavanger University College, the Norwegian Research Council, the National Science Foundation, the National Institute of Health, and the Norris Cotton Cancer Center (Dartmouth).

\section{REFERENCES}

Aronson BD, Johnson KA, and Dunlap JC (1994a) Circadian clock locus frequency: Protein encoded by a single open reading frame defines period length and temperature compensation. Proc Natl Acad Sci USA 91:7683-7687.

Aronson BD, Johnson KA, Loros JJ, and Dunlap JC (1994b) Negative feedback defining a circadian clock: Autoregulation of the clock gene frequency. Science 263:1578-1584.

Belozerskaya TA, Burikhanov SS, Chernyshova EK, Kritsky MS, and Lvov NP (1982) Does nitrate reductase play a key role in photoinduction of carotenoid synthesis in Neurospora crassa? Neurospora Newsletter 29:14-15.

Bünning E (1963) The Physiological Clock, Berlin, SpringerVerlag.

Cheng P, Yang Y, Wang L, He Q, and Liu Y (2003) WHITE COLLAR-1, a multifunctional neurospora protein involved in the circadian feedback loops, light sensing, and transcription repression of wc-2. J Biol Chem 278:3801-3808.

Collett MA, Garceau N, Dunlap JC, and Loros JJ (2002) Light and clock expression of the Neurospora clock gene frequency is differentially driven by but dependent on WHITE COLLAR-2. Genetics 160:149-158.

Correa A, Lewis ZA, Greene AV, March IJ, Gomer RH, and Bell-Pedersen D (2003) Multiple oscillators regulate circadian gene expression in Neurospora. Proc Natl Acad Sci USA 100:13597-13602.

Davis RH (2000) Neurospora: Contributions of a Model Organism, New York, Oxford University Press.

Deng M-D, Moureaux T, and Caboche M (1989) Tungstate, a molybdate analog inactivating nitrate reductase, deregulates the expression of the nitrate reductase structural gene. Plant Physiol 91:304-309.

Dunlap JC (1999) Molecular bases for circadian clocks. Cell 96:271-290.

Dunlap JC, Loros JJ, and DeCoursey PJ (2003) Biological Timekeeping, Sunderland, UK, Sinauer Associates.

Edmunds LN (1988) Cellular and Molecular Bases of Biological Clocks, New York, Springer-Verlag.

Field RJ and Burger M (1985) Oscillations and Traveling Waves in Chemical Systems, New York, John Wiley.

Froehlich AC, Liu Y, Loros JJ, and Dunlap JC (2002) White Collar-1, a circadian blue light photoreceptor, binding to the frequency promoter [see comment]. Science 297:815819.

Fu YH and Marzluf GA (1988) Metabolic control and autogenous regulation of nit-3, the nitrate reductase structural gene of Neurospora crassa. J Bacteriol 170:657661.

Garceau NY, Liu Y, Loros JJ, and Dunlap JC (1997) Alternative initiation of translation and time-specific phosphorylation yield multiple forms of the essential clock protein FREQUENCY. Cell 89:469-476.

Granshaw T, Tsukamoto M, and Brody S (2003) Circadian rhythms in Neurospora crassa: Farnesol or geraniol allow expression of rhythmicity in the otherwise arrhythmic strains frq10, wc-1, and wc-2. J Biol Rhythms 18:287-296.

Hall JC (1998) Genetics of biological rhythms in Drosophila. Adv Genet 38:135-184.

Kumar A and Paietta JV (1995) The sulfur controller-2 negative regulatory gene of Neurospora crassa encodes a protein with beta-transducin repeats. Proc Natl Acad Sci USA 92:3343-3347.

Lakin-Thomas PL and Brody S (2000) Circadian rhythms in Neurospora crassa: Lipid deficiencies restore robust rhythmicity to null frequency and white-collar mutants. Proc Natl Acad Sci USA 97:256-261.

Lakin-Thomas PL and Brody S (in press) Circadian rhythms in microorganisms: New complexities. Annu Rev Microbiol.

Lakin-Thomas PL, Cote GG, and Brody S (1990) Circadian rhythms in Neurospora crassa: Biochemistry and genetics. Crit Rev Microbiol 17:365-416.

Lee K, Dunlap JC, and Loros JJ (2003) Roles for WHITE COLLAR-1 in circadian and general photoperception in Neurospora crassa. Genetics 163:103-114.

Lillo C (1983) Studies of diurnal variations of nitrate reductase activity in barley leaves using various assay methods. Physiol Plant 57:357-362.

Lillo C (1984) Circadian rhythms of nitrate reductase activity in barley leaves. Physiol Plant 61:219-223.

Lillo C (1993) Light-induced circadian rhythms in $\mathrm{NADH}^{+}$glyceraldehyde-3-phosphate dehydrogenase mRNA in corn seedlings. J Interdiscipl Cycle Res 24:65-71.

Lillo C, Meyer C, and Ruoff P (2001) The nitrate reductase circadian system: The central clock dogma contra multiple oscillatory feedback loops. Plant Physiol 125:15541557.

Lillo C and Ruoff P (1989) An unusually rapid light-induced nitrate reductase mRNA pulse and circadian oscillations. Naturwissenschaften 76:526-528.

Loros JJ and Dunlap JC (2001) Genetic and molecular analysis of circadian rhythms in Neurospora. EMBO J 20:109117.

Loros JJ and Feldman JF (1986) Loss of temperature compensation of circadian period length in the frq-9 mutant of Neurospora crassa. J Biol Rhythms 1:187-198.

Marzluf GA (1997) Genetic regulation of nitrogen metabolism in the fungi. Microbiol Mol Biol Rev 61:17-32.

McClung CR (2001) Circadian rhythms in plants. Annu Rev Plant Physiol Plant Mol Biol 52:139-162.

McClung CR and Kay SA (1994) Circadian rhythms in Arabidopsis thaliana. In Arabidopsis thaliana, Somerville, 
CR, Meyerowitz, E. eds, pp 615-637, Cold Spring Harbor, NY, Cold Spring Harbor Press.

McWatters H, Dunlap JC, and Millar AJ (1999) Circadian biology: Clocks for the real world. Curr Biol 9: R633-R635.

Merrow M, Brunner M, and Roenneberg T (1999) Assignment of circadian function for the Neurospora clock gene frequency. Nature 399: 584-586.

Nakashima H and Onai K (1996) The circadian conidiation rhythm in Neurospora crassa. Semin Cell Dev Biol 7:765774 .

Ninnemann H (1997) Cooperative blue/UV-light absorbing pigments in fungal and plant photoreception. In Modern Topics in Photochemistry and Photobiology, Vargas, F, ed, pp 115-154, Trivandrum, India, Research Signpost.

Ninnemann H (2001) Developments in blue light reception/ signal transduction in fungal morphogenesis: Neurospora/Phycomyces, nitrate reductase and NOsynthase. Recent Res Devel Plant Biol 1:105-130.

Nowrousian M, Duffield GE, Loros JJ, and Dunlap JC (2003) The frequency gene is required for temperature-dependent regulation of many clock-controlled genes in Neurospora crassa. Genetics 164:923-933.

Okamoto PM, Fu YH, and Marzluf GA (1991) Nit-3, the structural gene of nitrate reductase in Neurospora crassa: Nucleotide sequence and regulation of mRNA synthesis and turnover. Mol Gen Genet 227:213-223.

Pan H, Feng B, and Marzluf GA (1997) Two distinct proteinprotein interactions between the NIT2 and NMR regulatory proteins are required to establish nitrogen metabolite repression in Neurospora crassa. Mol Microbiol 26:721729.
Perkins DD, Radford A, and Sachs MS (2001) The Neurospora Compendium, San Diego, Academic Press.

Premakumar R, Sorger GJ, and Gooden D (1979) Nitrogen metabolite repression of nitrate reductase in Neurospora crassa. J Bacteriol 137:1119-1126.

Roenneberg T and Rehman J (1996) Nitrate, a nonphotic signal for the circadian system. FASEB J 10:1443-1447.

Roldán JM and Butler WL (1980) Photoactivation of nitrate reductase from Neurospora crassa. Photochem Photobiol 32:375-381.

Ruoff P, Behzadi A, Hauglid M, Vinsjevik M, and Havas H (2000) $\mathrm{pH}$ homeostasis of the circadian sporulation rhythm in clock mutants of Neurospora crassa. Chronobiol Int 17:733-750.

Ruoff P, Vinsjevik M, Monnerjahn C, and Rensing L (1999) The Goodwin oscillator: On the importance of degradation reactions in the circadian clock. J Biol Rhythms 14:469-479.

Sorger GJ, Premakumar R, and Gooden D (1978) Demonstration in vitro of two intracellular inactivators of nitrate reductase from Neurospora. Biochim Biophys Acta 540:3347.

Vogel HA (1956) A convenient growth medium for Neurospora (medium N). Microbiol Gen Bull 15:42-43.

Walls S, Sorger GJ, Gooden D, and Klein V (1978) The regulation of the decay of nitrate reductase: Evidence for the existence of at least two mechanisms of decay. Biochim Biophys Acta 540:24-32.

Young MW (1998) The molecular control of circadian behavioral rhythms and their entrainment in Drosophila. Annu Rev Biochem 67:135-152. 